\title{
IMPACT OF COSMIC RADIATION ON AVIATION RELIABILITY AND SAFETY
}

\author{
Ian Zaczyk* \\ Mirce Akademy, Woodbury Park, Exeter, United Kingdom
}

The main objective of this paper is to argue that the scientific approach to reliability and safety is the only way forward for the reliability community if accurate predictions regarding occurrences of negative functionability events are to be made and subsequently verified during the operational processes of the future man made, managed and maintained systems. For that to happen, a scientific understanding of the mechanisms that cause occurrences of functionability events of the surrounding natural environment are required. Then and only then, can accurate and meaningful reliability and safety predictions become possible, enabling the ultimate goal of reducing the probability of failure event occurrences during the life of man made, managed and maintained systems. This paper focuses on the scientific understandings of the relevant cosmic radiation on aviation reliability and safety. Key words: Aviation, Cosmic radiation, Functions, Predictions, Event, Reliability, Safety

\section{INTRODUCTION}

Due to the rapid advances in electronics technology and the unrelenting demand for increased avionics functionality in the competitive commercial aircraft industry, the complexity of avionics systems has risen exponentially. As a consequence, ever more advanced microprocessor and memory semiconductor devices are being used that exhibit an increased susceptibility to cosmic radiation phenomena.

Single Event Effects (SEEs) have been the primary radiation concern for avionics since the late 1980's when the phenomenon, which had previously only been observed in orbiting satellites, also began to appear in aircraft electronic systems. According to Baumann, "Left unchallenged, soft errors have the potential for inducing the highest failure rate of all other reliability mechanisms combined" [01]. Atmospheric radiation causes daily concerns regarding the reliability and safety of avionics equipment, particularly for those systems that are considered safety.

The trend with each new generation of avionics system is to use increasing quantities of semiconductor memories and other complex devices that are susceptible to failures induced by ionising radiation from the following two main sources: cosmic rays from space and alpha particles from radioactive impurities in the device itself. The interaction of this radiation can result in ei- ther a transient 'soft error' effect such as a bit flip in memory or a voltage transient in logic, alternatively a 'hard error' can be induced resulting in permanent damage such as the burn out of a transistor. These functionability effects caused by a single radiation event are collectively termed as Single Event Effects (SEEs).

If device memory cells used for flight safety or mission critical functions are affected the concern is that the loss of key system functionality due to corrupted data could cause a flight safety or mission critical failure. The ability to predict and quantify the rate of occurrence of erroneous data bits in memories or voltage transients in logic is one of the key objectives in the field of avionics SEEs research.

The main challenge of in-service operation of man made and maintained systems, is the true understanding of the impact of the environment that surrounds them. The reliability and safety of their operation is influenced by a multitude of different factors extending from the Earth's atmosphere to the far reaches of space beyond our own galaxy. In order to determine the probabilities of occurrence and the resultant impact of functionability events on a system a full awareness of the dynamic nature of the environmental phenomena is required. To identify the causes of functionability events the mechanisms that cause those physical phenomena must first be understood [02]. 
Consequently, the main objective of this paper is to argue that the scientific approach to reliability and safety is the only way forward for all members of the reliability community who wish to make accurate predictions regarding occurrences of negative functionability events, which will be confirmed during the operational processes of the future systems. For that to happen a scientific understanding of functionability phenomena is required. This paper advocates that research of this nature must include the understanding of the cosmic radiation phenomena, in order for the occurrence of functionability events to be understood. Then and only then, can accurate and meaningful reliability and safety predictions become possible, enabling system design and operational changes to be made to reduce the probability of failure event occurrences during the life of man made, managed and maintained systems.

\section{SCIENTIFIC PRINCIPLES OF MIRCE MECHANICS}

Mirce Mechanics is a new scientific theory, developed at the MIRCE Akademy by Dr. J. Knezevic, that aims to scientifically understand the physical causes and human actions that shape the motion of functionability through lives of man made, managed and maintained systems [02]. For years, research studies, international conferences, summer schools and other events have been organised in order to explain and draw attenton to just the physical scale at which failure phenomena should be studied and understood. In order to understand the motion of functionability events it is necessary to understand the physical mechanisms that cause their occurrences. That represented a real challenge, as the answers to the question "what are the discrete physical and chemical processes that lead to the occurrence of given functionability events" have to be determined. Without accurate answers to those questions the prediction of their future occurrences is not possible, and without ability to predict the future, the use of the word science becomes inappropriate.

After a numerous discussions, studies and trials, it has been concluded that any serious studies in this direction, from Mirce Mechanics point of view, have to be based between the following two boundaries:

- The "bottom end" of the physical world, which is at the level of the atoms and molecules that exists in the region of 10-10 of a metre,

- The "top end" of the physical world, which is at the level of the solar system that stretches in the physical scale around $10+10$ of a metre.

This range is the minimum sufficient "physical scale" which enables scientific understanding of relationships between system life processes and system failure events.

One of the interacting factors from the physical world that directly impacts the functionability trajectory of man made systems are cosmic phenomena, as illustrated by the examples given above. This paper therefore considers major causes of cosmic phenomena from the physical world that can influence system functionability from a reliability and safety point of view.

To achieve this goal, this paper examines the cosmic phenomena to understand their mechanisms of occurrence; describes the potential negative functionability event processes that can be initiated and their possible impacts on aviation systems reliability and safety.

\section{ATMOSPHERIC RADIATION}

In the natural environment there are two fundamental radiation particles that can cause transient errors in electronic devices, which can be classified into the following three groups:

I. High-energy cosmic ray neutrons.

II. Thermal or low energy cosmic ray neutrons.

III. Low energy alpha particles emitted from within the semiconductor device and packaging materials.

Each of these particle categories is different in terms of flux, energy level, charge or composition, but in essence a single particle of any of the above forms could result in a soft error if it deposits sufficient charge within the susceptible volume of a device.

\section{COSMIC RAYS}

Cosmic rays are individual energetic particles that originate from a variety of energetic sources ranging from our Sun to supernovas and other phenomena in distant galaxies all the way out to the edge of the visible universe. The majority of energetic particles however come from our galaxy with only the most energetic particles believed to 
have originated from extra-galactic sources. Although the term cosmic ray is commonly used, this term is misleading because no cohesive ray or beam actually exists. Cosmic rays are in fact independent energetic particles that travel at approximately $87 \%$ of the speed of light.

Victor Hess first discovered cosmic rays in 1912, when he discovered the fourfold increase in ionisation rates as he ascended to altitude in a balloon. From this experiment he concluded that "the results of my observation are best explained by the assumption that a radiation of very great penetrating power enters our atmosphere from above." In 1936 he was awarded the Nobel Prize in Physics for this discovery, although the term 'cosmic rays' is actually credited to a fellow scientist, R.A Millikan in 1925.

The majority of cosmic rays consist of the nuclei of atoms (atoms stripped of their outer electrons) ranging from the lightest elements in the periodic table to the heaviest. In terms of composition about $90 \%$ of the nuclei are hydrogen, therefore just single protons, $9 \%$ are helium, alpha particles with the remaining $1 \%$ a mix of heavier element nuclei, high energy electrons, positrons and other sub-atomic particles.

Cosmic rays must not be confused with gamma rays (high energy photons) that constitute the most energetic form of electromagnetic radiation. However there is a component of cosmic rays, $<0.1 \%$ which consists of gamma ray photons produced after high energy particle collisions with matter.

Within the atmosphere the three most important parameters used to define the variability of the particle flux at a specific location are altitude, latitude and energy. Within the field of cosmic ray physics altitude is expressed in terms of atmospheric depth, which is the mass thickness per unit of area in the Earth's atmosphere. At sea level this is approximately $1033 \mathrm{~g} / \mathrm{cm} 2$ of oxygen and nitrogen and reduces as the altitude increases. Atmospheric depth is the key-determining factor in the particle flux for a specific point in the atmosphere. For example at an altitude of $3000 \mathrm{~m}$ the flux of neutrons within the atmospheric cascade is around 10 times greater than at sea level.

Energy is usually shown as the flux per unit of energy called the differential flux, and geographic latitude is expressed in terms of the geomagnetic field strength expressed in units of $\mathrm{GeV}$ and also referred to as a locations geomagnetic rigidity or cut-off.

Cosmic rays can be broadly divided into two main categories, primary cosmic rays and secondary cosmic rays. Primary cosmic rays are particles accelerated at astrophysical sources and generally do not penetrate the Earth's atmosphere. Primary cosmic rays are composed from a mixture of different energetic particles that can be categorised based on origin and energy level into the groups listed below in order of descending particle energy:

I. Extra galactic cosmic rays,

II. Galactic cosmic rays,

III. Solar cosmic rays,

IV. Anomalous cosmic rays.

As cosmic ray particles are charged, magnetic fields in space will bend their motion paths. Due to the impact of magnetic fields, cosmic ray particles are incident on the Earth from all directions and as a consequence it is impossible to retrace their trajectories to determine their point of origin. However, the trajectory of a gamma ray photon is a straight line, due to their neutral charge. This makes it possible to retrace the trajectories of gamma rays to discover their source.

\section{Extra galactic and galactic cosmic rays}

Extra galactic cosmic rays originating from outside our galaxy and galactic cosmic rays from within bombard the top of the Earth's atmosphere with a low but continuous flux of protons and heavy ions. The majority of energetic particles are accelerated from within our galaxy but external to the solar system. Cosmic ray particles from extra galactic and galactic sources are typically highly energetic and arrive at the Earth with an approximate flux rate of between 2 to 4 $\mathrm{cm}^{-2} \mathrm{~s}^{-1}$.

\section{Solar cosmic rays}

Solar cosmic rays, also termed Solar Energetic Particles, SEPs or Solar Proton Events SPEs, are produced by highly energetic processes that occur on or close to the Sun's surface. Unlike galactic cosmic rays that arrive at the Earth with an almost steady constant flux, the occurrence of solar particles is not only irregular but also highly variable in terms of flux rate. Typically most solar protons arriving from the Sun lack the energy level required to penetrate the Earth's magnetic field. 
Solar cosmic rays consist of heavy ions and protons with a less energetic spectrum than galactic cosmic rays. In comparison to the maximum energy possessed by galactic cosmic ray protons of $10^{21} \mathrm{eV}$, the solar proton peak energy of about $20 \mathrm{GeV}$ is many orders of magnitude smaller.

In the case of very powerful flux ejections, SPEs manifest as Ground Level Enhancements or Events, GLEs, on the Earth's surface and typically last between 20 minutes to a few days dependent on the originating solar mechanism. SPEs can therefore be categorised as either an impulsive event linked to solar flares or gradual events linked to coronal mass ejections, CMEs. The main concern however regarding SPEs are the significant neutron flux enhancements generated at aircraft altitudes particularly at high geographic latitudes where the Earth's level of magnetic shielding is reduced.

During the Sun's eleven year solar cycle the flux of solar particles incident upon the Earth's upper atmosphere can increase by a million fold during a GLE relative to the level at a quiescent period close to or at the solar minimum. In contrast the difference between the flux rates between solar minimum and solar maximum, whilst still significant, are less dramatic than the sporadic peak flux rates caused by the most energetic SPEs, as shown in Table 1.

Table 1: Mean integral solar cosmic ray flux at solar minimum and maximum

\begin{tabular}{|c|c|c|}
\hline $\begin{array}{c}\text { Energy } \\
\text { Range }\end{array}$ & $\begin{array}{c}\text { Solar Maximum } \\
\left(\text { Particles: } \mathrm{cm}^{-2} \mathrm{~s}^{-1}\right)\end{array}$ & $\begin{array}{c}\text { Solar Minimum } \\
\left(\text { Particles: } \mathrm{cm}^{-2} \mathrm{~s}^{-1}\right)\end{array}$ \\
\hline $\begin{array}{c}\text { Above } \\
30 \mathrm{MeV}\end{array}$ & $3 \times 10^{2}$ & $2 \times 10^{-2}$ \\
\hline $\begin{array}{c}\text { Above } \\
100 \mathrm{MeV}\end{array}$ & 20 & $2 \times 10^{-3}$ \\
\hline
\end{tabular}

GLEs in general occur 1 to 3 years after a solar maximum and to date since 1942 in total 71 of them have been observed. Over a longer period analysis of nitrate spikes obtained from polar ice cores indicate 154 large SPEs have occurred in the last 450 years. These powerful and evidently rare events are believed to be caused by the most energetic solar flares rather than CMEs.

In terms of energy levels SPEs typically range from $10 \mathrm{MeV}$ to $100 \mathrm{MeV}$ although protons up to $20 \mathrm{GeV}$ travelling at near relativistic speeds can be discharged from the Sun during extremely energetic events. The proton energy level determines the speed and hence the arrival time of incident protons. At $1 \mathrm{MeV}$, protons arrive in 2.9 hrs but at $1 \mathrm{GeV}$ the arrival time is reduced to just 9.5 minutes.

\section{Anomalous cosmic rays}

Anomalous cosmic rays are the final component of primary cosmic rays and possess energy levels significantly lower than any other type of cosmic ray, typically less than $\sim 10 \mathrm{MeV}$. They are created when electrically neutral atoms enter the heliosheath of the Sun's solar wind, become ionised and are then accelerated by the termination shock. The termination shock region forms the inner edge of the heliosheath where the solar wind becomes subsonic. This region varies between 75 and $100 \mathrm{AU}$ ( $1 \mathrm{AU}$ is a unit of length approximately equal to the semi-major axis of Earth's orbit around the Sun) from the Earth.

\section{SINGLE EVENT EFFECTS IN AVIONICS}

Single Event Effects (SEEs) have been the primary radiation concern for avionics since the late 1980's when the phenomenon, which had previously only been observed in orbiting satellites, also began to appear in aircraft electronic systems.

The principal SEE affecting avionic devices is the Single Event Upset (SEU) caused when a sole incident particle creates a charge disturbance of sufficient magnitude in a memory cell, flip-flop, latch or register to reverse or flip its currently stored data state. Alternatively, in logic or support circuitry a transient voltage pulse can be generated that dependent on the right conditions can propagate through the logic of the device and become latched into a memory cell. Voltage spikes on power supply lines and noise can also cause transient errors; however appropriate shielding and filtering design measures can suppress these types of disturbances.

The primary sources of radiation are high energy cosmic particles, low energy (thermal) neutrons and low energy alpha particles emitted from device and packaging contaminants.

Radiation can affect electronic devices as the consequence of a single energetic particle strike, termed 'single event' or as multiple strikes over an extended period of time. The effects due to multiple events, Total Ionisation Dose (TID) and displacement damage manifest gradually in electronic components as damage is accumulated over time. These total dose effects and hard 
SEEs whilst relevant to electronic systems operating in the harsher space environment have a negligible effect on current semiconductor devices used in the terrestrial environment.

Whilst each form of SEE is considered in this paper the main focus will be on SEUs which are the dominant device negative functionability event mechanism affecting electronic devices in the avionics environment.

The second most prevalent SEE is the Multiple Bit Upset (MBU) that occurs when a single particle causes the upset of two or more memory cells. Fortunately MBUs only form a fraction of the total number of SEUs, hence they have little significance except for memory architectures employing Error Detection and Correction, (EDAC) techniques. In these circumstances, dependent on the type of error correction technique employed, multiple bit errors could have significant consequences if the protected memory is used for flight or mission critical applications. MBUs are generally assumed to attribute $3 \%$ of the total upset rate [04] although rates as high as 5\% have been reported.

Following MBU, Single Event Functional Interrupt (SEFI) and Single Event Latch ups (SEL) account for the majority of the remaining proportion of SEEs affecting avionic devices. SEFIs occur when an upset initiates an IC test mode or reset mode that causes the device to temporary lose functionality. SELs arise when an incident particle creates a charge disruption sufficient enough to effectively short circuit the device resulting in its permanent change of state or in some circumstances permanent damage if excessive current flows as a result of the latch-up.

The last SEE of avionics relevance that can generate soft errors in the core logic of microprocessors and microcontrollers is the Single Event Transient (SET). They are transient and non-destructive in nature and are capable of producing a soft error, (i.e. the storage of an erroneous data value in registers, memories or latches) only if it is propagated through the logic pathways of the device. This is dependent on the dynamic state of the logic at the time of the particle induced nodal voltage transition and the configuration of the logic pathways within the device. If a soft error occurs normal system behaviour can be restored by resetting or rewriting the incorrect data.

Of all the forms of SEE, SEUs are the most prevalent in avionic electronic devices; Table 2 illus- trates the approximate distribution percentage values, between each type of SEE except SETs, for which no reliable data exists. This problem of limited SEE statistical data is an enduring problem in the field as the capturing and recording of SEEs during flight is impeded by:

I) Fault tolerant designs and error correction techniques.

II) SEEs incorrectly diagnosed as electrical interference or random component negative functionability events.

III) Reluctance of semi-conductor manufactures to disclose proprietary information regarding the root cause negative functionability event mechanisms and the historic negative functionability event statistics gathered from devices returned from in-flight usage.

Table 2: Main SEE Apportionments - Avionics

Environment

\begin{tabular}{|c|c|}
\hline Single Effect Event Type & Percentage \\
\hline Single Event Upset & $90 \%$ \\
\hline Multiple BIT Upset & $5 \%$ \\
\hline Single Event Functional Interrupt & $3 \%$ \\
\hline Single Event Latchup & $2 \%$ \\
\hline
\end{tabular}

The current convention is to discuss the rate of SEU occurrence in terms of soft error rates (SER), which are measured in failures in time, (FIT). One failure in 1 billion device operating hours is defined as $1 \mathrm{FIT}$. This term is also widely used in the semiconductor industry to state the expected occurrence rate of hard negative functionability event mechanisms.

The first efforts to calculate SEU rates were presented in two papers in 1984, Tsao et al. [05] and a companion paper by Silberberg et al. [06]. The Tsao paper detailed methods of calculating SEU rates from primary \& secondary cosmic rays reaching down to $40,000 \mathrm{ft}$ and the Silberberg paper introduced methods for calculating SEU rates resulting from secondary neutrons in the atmosphere.

The reason that semiconductors have become susceptible to SEEs in the terrestrial environment rather than existing solely in space can be partially attributed to the commercial demands for increased functionality and performance, whilst lowering power consumption and cost. To fulfil these requirements component manufactures have continued to reduce the geometry size of 
integrated circuits with each new generation resulting in higher gate speeds, increased feature density and reduced power consumption.

Whilst technology scaling enables the demands of system designers to be met the downside of this is an increased sensitivity to radiation. Within a memory device this is caused by a reduction in the capacitance inside a cell and a significant increase in the number of cells that could potentially be upset within each device. Less capacitance in a device due to the shrinking of process technology and reduced supply voltage means that the minimum amount of charge necessary to hold data in a device, either a logic 1 or 0 is also reduced. This quantity of charge, known as the critical charge, is therefore more susceptible to a charge disturbance caused by an incident radiation particle, thus eroding the components resistance to SEUs. The approximate critical charge of a node can be calculated using the expression:

$$
Q_{c}=C_{\text {node }} \times V D D
$$

where: $Q_{c}$ is Critical Charge, $C_{\text {node }}$ is Node Capacitance and $V D D$ is Operating Voltage.

A lower nodal critical charge is therefore more likely to be ' upset ' by incident particles with a lower energy, because the flux of energetic particles increases at lower energy levels.

The components most susceptible to SEU are therefore devices that contain the largest number and density of potentially volatile bits namely memories and microprocessors. Table 3 contains a list of the devices that are currently considered to be the most susceptible to SEU in aircraft avionics systems and includes the specific regions within the architecture that are most at risk.

Table 3: SEU Sensitive Devices

\begin{tabular}{|c|c|}
\hline Devise Type & Sensitive Areas \\
\hline SRAMS and DRAMS & $\begin{array}{c}\text { Memory cells } \\
\text { and control logic }\end{array}$ \\
\hline $\begin{array}{c}\text { Microprocessors and } \\
\text { Microcontrollers }\end{array}$ & $\begin{array}{c}\text { Registers, cache, } \\
\text { sequential and } \\
\text { combinational control } \\
\text { logic }\end{array}$ \\
\hline \hline FPGAs and ASICs & $\begin{array}{c}\text { Combinatorial logic and } \\
\text { sequential logic }\end{array}$ \\
\hline
\end{tabular}

Opto-electronics and power switching components are also susceptible, to various forms of hard and soft SEE but are not considered in this paper due to their very low probability of failure in the avionics radiation environment.

Each of the factors discussed in this section, increased functionality and performance, lack of specialist devices, lower critical charge and higher cell density all impact upon the SEU tolerance of advancing semiconductor designs.

The net effect is an increase in the overall device SER that if not adequately mitigated against using appropriate methods such as error detection and correction (EDAC) and architectural redundancy, will result in an increased system SER, plus potentially an increase in the number of mission or flight safety critical negative functionability events.

\section{CONCLUSION}

The main objective of this paper was to demonstrate the necessity of addressing all physical causes that lead to the transition of a system from positive to negative functionability state during its life. Addressing the reliability and safety characteristics of a system in isolation from the investigation of the impact of the natural environment is not sufficient. Hence, results of the research performed in [04], presented here; have shown the significant impact of cosmic radiation on the occurrence of negative functionability events, and consequently, the necessity for addressing them when considering the reliability and safety characteristics of avionics, at the design stages of a systems development.

This paper presents the research results obtained by applying the principles of Mirce Mechanics to the scientific understanding of the physical mechanisms that lead to the occurrence of the Single Event Upset (SEU), which is the principal SEE affecting avionic devices. It is caused when a sole incident particle creates a charge disturbance of sufficient magnitude in a memory cell, flip-flop, latch or register to reverse or flip its currently stored data state. Alternatively, in logic or support circuitry a transient voltage pulse can be generated that dependent on the right conditions can propagate through the logic of the device and become latched into a memory cell.

In summary this paper advocates that any system reliability and safety considerations must include the full understanding of the complex interactions between functionability significant processes and to determine the influence of each discrete factor, on the functionability trajectory 
through life of a complete avionics system. Then and only then, can accurate and meaningful reliability and safety predictions become possible, enabling the ultimate goal of reducing the probability of the occurrence of negative functionability events during the life of man made, managed and maintained systems.

\section{ACKNOWLEDGEMENT}

Authors wish that acknowledge the financial support obtained from the Research Fund of the MIRCE Akademy that enabled this research to be performed.

\section{REFERENCES}

1) Baumann, R., " Radiation-induced soft errors in advanced semiconductor technologies, " IEEE Transactions on Device and Materials Reliability, vol 5, No 3, pp. 305-316, Sept. 2005.

2) Knezevic, J. Time To Choose between Scientific and Administrative Approach to Reliability, Journal of Applied Engineering Science, No 3,. Year 2012, Vol 10. Belgrade, Serbia.

3) Knezevic, J/, Atoms and Molecules in Mirce Mechanics Approach to Reliability, SRESA Journal of Life Cycle Reliability and Safety Engineering, Vol 1, Issue 1, pp 15-25, Mumbai, India, 2012. ISSN-22500820

4) Zaczyk. I, "Analysis of the Influence of Atmospheric Radiation Induced Single Event Effects on Avionics Failures", Master Dissertation, MIRCE Akademy, Exeter, UK, 2010.

5) C. H. Tsao, R. Silberberg, and J. R. Letaw, "Cosmic ray heavy ions at and above 40,000 feet, "IEEE Trans. Nucl. Sci., vol. 31, pp. 1066-1068, Dec 1984.

6) R. Silberberg, C. H. Tsao, and J. R Letaw, "Neutron generated single event upsets," IEEE Trans. Nucl. Sci., vol. 31, pp. 11831185, Dec 1984.

Paper sent to revision: 14.11.2013.

Paper ready for publication: 11.12.2013. 